\title{
RECURRENCE-PARTITIONS OF FINITE MEASURE SPACES WITH APPLICATIONS TO ERGODIC THEORY
}

\author{
BY
}

\author{
RUDOLPH W. PREISENDORFER AND BERNARD W. ROOS
}

Introduction. The object of this note is to develop and apply a certain class of measure-theoretic partitions induced by invertible measure-preserving transformations on finite measure spaces. The utility of such partitions is illustrated by the following examples which are considered, with others, in detail below: (a) a generalization of Kac's theorem on the integral of the recurrence function over a measurable set; (b) some integral theorems for the exit function, the natural complement of the recurrence function; and (c) a special criterion for the ergodicity of invertible measure-preserving transformations on finite measure spaces which may conceivably be of physical interest.

The concept of an invertible measure-preserving transformation, as it is used today in modern measure theory, is a mathematical abstraction traceable to its well-known physical origin in the study of phase-space representations of conservative mechanical systems. In particular, it stems from Liouville's throrem on the conservation of the phase-space measure $\mu$ under an invertibl: transformation $\phi_{t}: \mu\left(\phi_{t}^{-1}(E)\right)=\mu(E)$, where $E$ is any measurable subset of phase-space, $\mu$ its usual Lebesgue measure, and $\phi_{t}$ the phase map indexed by time $t$, which is induced by the (generally unique) solution of the Hamiltonian formulation of the phase problem. More recent physical examples of measure-preserving transformations which give further evidence of the enduring potential interest in these objects may be cited: the transport equations for photons and neutrons streaming in vacua give rise to measurepreserving transformations. The measures which are conserved in these instances are "optical volumes" of subsets of the respective carrier spaces. Further examples arise in fluid-dynamic studies-in both mechanical and electromagnetic contexts.

Preliminary definitions. A measurable space is an ordered pair $(X, \mathfrak{S})$ where $X$ is a set and $\subseteq$ a $\sigma$-ring of subsets (the measurable sets) of $X$ (i.e., a nonempty class of sets closed under set-difference and countable set union operations). A partition of a measurable space $(X, \mathbb{S})$ is a countable (possibly finite) class of disjoint elements of $\subseteq$ whose union is $X$. A measure space is an ordered triple $(X, \mathfrak{S}, \mu)$ where $(X, \mathfrak{S})$ is a measurable space and $\mu$ is a measure on $\mathfrak{S}$ (i.e., $\mu$ is a real valued, nonnegative, countably additive function on $\mathbb{S}$, with $\mu(\phi)=0$ ). In all that follows $\mathfrak{S}$ will be a $\sigma$-algebra, i.e., a $\sigma$-ring with $X \in \mathfrak{S}$. A measure space is finite if $\mu(X)<\infty$; in this case $\mu$ may be normalized: $\mu(X)=1$. A measurable set $E$ is positive iff (if and only if) $\mu(E)>0 . E$ is a

Received by the editors August 15, 1960. 
(measurably) proper subset of $F$ if $E \subset F$ and $\mu(E)<\mu(F), E, F \in \mathfrak{S}$.

All transformations below will have as domain and range the set $X$ of a given measurable space. A transformation $T$ is measurable iff $T^{-1}(E) \in \mathfrak{S}$ whenever $E \in \mathfrak{S}$. A measurable transformation $T$ is measurability-preserving iff $T(E) \in \mathfrak{S}$ whenever $E \in \mathfrak{S}$; and iff $T$ is, in addition, one-to-one and onto, it is invertible. A measurable transformation $T$ on $(X, \mathfrak{S}, \mu)$ is measurepreserving iff $\mu\left(T^{-1}(E)\right)=\mu(E)$ for every $E \in \subseteq$. It should be noted.that a measure-preserving transformation need not be measurability-preserving. In fact, examples exist [2] which show that, as the notions are defined here, a transformation may be one-to-one, onto, and measure-preserving and yet not invertible (by not having $T^{-1}$ measurable).

A set $E \subset X$ is invariant under a transformation $T$ if $T^{-1} E=E$. $F \subset E$ is recurrent in $E$ iff there exists an integer $k$ such that $T^{-k} F \subset E$. $F \subset E$ is said to have a recurrent part (with respect to $E$ ) iff $F$ has a positive subset recurrent in $E$. A measurable transformation $T$ on $(X, \mathfrak{S}, \mu)$ is ergodic iff it has only trivial invariant subsets, i.e., iff either $\mu(E)=0$ or $\mu\left(E^{\prime}\right)=0$ whenever $E \in \subseteq$ is invariant under $T$, where $E^{\prime}=X-E$. By the preceding comments and definitions it should be clear that the notions of ergodicity, invertibility, and measure-preservation are pairwise independent. Finally, in all that follows, for a given transformation $T$, let $T^{n}$, the $n$th iterate of $T, n \geqq 1$, be defined inductively as: $T^{n}=T^{n-1} T$, where $T^{1}=T$; and set $T^{0}=I$, the identity transformation.

Recurrence and exit functions. The recurrence function (or Poincaré cycle) used by $\mathrm{Kac}$ [1] in essence gives the "least time" required for any point, which is propelled by a transformation on a space, to return to some given set. It is this function which was of interest in Kac's theorem and which at present forms the point of departure for the study of the recurrencepartition of a general measure space:

DEFINITION. Let $T$ be a measurable transformation on a measurable sface $(X, \mathfrak{S})$. Let $E \in \mathfrak{S}$, and let $n(\cdot, E)$ be a function on $E$ with range in the measurable space of positive integers such that, for each $E, n(x, E)$ is the least positive integer $n$ with the property:

$$
T^{n} x \in E .
$$

$n(\cdot, E)$ is the recurrence function on $E$.

If for some $x \in E, n(x, E)$ does not exist, we say that $n(\cdot, E)$ is not defined at $x$. In what follows, $n(\cdot, E)$ always will be assumed to be defined on a given measure space $(X, \mathfrak{S}, \mu)$ as follows: we shall always require $n(\cdot, E)$ to be defined everywhere $[\mu]$ on $E \in \subseteq$. ( ${ }^{(1)}$ Further, to avoid certain inessential pathologies, we establish the convention that $n(x, E)=0$ for $x \in E^{\prime}$, thereby extending in a trivial and harmless way the domain of definition of all recurrence

(1) Here and below a bracketed measure symbol means that the statement just before it is true except for possibly a set of $\mu$-measure zero in a given measure space $(X, \mathfrak{S}, \mu)$. 
(and exit functions below) to the whole space $X$. The physical significance of the nondefinition of $n(x, E)$, where $x \in E$, is that the images of $x$ under all positive interates of $T$ lie outside $E$.

A natural companion of the recurrence function $n(\cdot, E)$ is the exit function. Whereas $n(\cdot, E)$ gives the "least time" of return to a point of $E$, the exit function gives the "least time" of exit or departure from $E$ :

DEFINITION. Let $T$ be a measurable transformation on a measurable space $(X, \mathfrak{S})$. Let $E \in \subseteq$, and let $m(\cdot, E)$ be a function on $E$ with range in the measurable space of positive integers such that, for each $x \in E, m(x, E)$ is the least positive integer $m$ with the property:

$$
T^{m} x \in E^{\prime} .
$$

$m(\cdot, E)$ is the exit function on $E$.

If for some $x \in E, m(x, E)$ does not exist, then $m(\cdot, E)$ is not defined at $x$. The definition conventions for $m(\cdot, E)$ exactly parallel those of $n(\cdot, E)$. In particular, the physical interpretation of the nondefinition of $m(\cdot, E)$ is that the images of $x$ under all positive iterates of $T$ lie inside $E$.

The study of the recurrence and exit functions is facilitated by determining the sets over which they are constant-valued. These sets in turn form the basis for the class of partitions of the space $\left(X, S_{,} \mu\right)$ of interest in the present work. Thus, in the case of $n(\cdot, E)$, set:

$$
E_{1}=\{x: x \in E, T x \in E\}
$$

and for $n>1$,

$$
E_{n}=\left\{x: x \in E, T^{n} x \in E, T^{i} x \in E^{\prime}, 1 \leqq j<n\right\} .
$$

Hence

$$
n\left(\left\{E_{j}\right\}, E\right)=j, \quad j=1,2, \cdots .
$$

Suppose the sets $E_{j}$ are measurable. Then since the $\{j\}$ are (singleton) Borel sets of the measurable space of positive integers, we have

$$
E_{j}=n^{-1}(\cdot, E)(\{j\}),
$$

so that $n(\cdot, E)$ would clearly be measurable. To show that the $E_{j}$ are measurable, it is sufficient to observe that

$$
E_{1}=T^{-1} E \cap E
$$

and that, for $n>1$ :

$$
E_{n}=\left[\bigcap_{j=1}^{n-1}\left(T^{-i} E\right)^{\prime}\right] \cap T^{-n} E \cap E .
$$

Since $T$ is measurable, it follows that the sets $T^{-n} E$ are measurable for all $n \geqq 1$. Further, $\subseteq$ is closed in particular under the operations of intersection 
and complementation. Hence, by (5) and (6) $E_{j} \in S$ for $j \geqq 1$. This proves

LEMMA 1. The recurrence function $n(\cdot, E)$ is measurable $[\mu]$ on $E \in \mathbb{S}$.

In a similar way the measurability of $m(\cdot, E)$ is demonstrated by first setting

$$
D_{1}=T^{-1} E^{\prime} \cap E
$$

and by defining, for $n>1$ :

$$
D_{n}=\left[\bigcap_{j=1}^{n-1}\left(T^{-i} E^{\prime}\right)^{\prime}\right] \cap T^{-n} E^{\prime} \cap E .
$$

The elements of the class $\mathfrak{D}=\left\{D_{j}: j=1,2, \cdots\right\}$ are evidently the sets over which $m(\cdot, E)$ is constant-valued with the value $m(x, E)=j$ for $x \in D_{j}$. Repeating the arguments given above, now for $m(\cdot, E)$, yields

Lemma 2. The exit function $m(\cdot, E)$ is measurable $[\mu]$ on $E \in S$.

The pertinent analytical connection between the recurrence and exit functions is given by

Lemma 3. If $E \in S$, then $n(\cdot, E)=\chi_{E-E_{1}} m\left(T(\cdot), E^{\prime}\right)+1 \quad[\mu]$.

Proof. If $x \in E_{1} \subset E$, then the characteristic function $\chi_{E-E_{1}}$ of the set $E-E_{1}$ has value $\chi_{E-E_{1}}(x)=0$, so that $n(x, E)=1$. On the other hand if $x \in E-E_{1}$, $\chi_{E-B_{1}}(x)=1$ and $n(x, E)=m\left(T x, E^{\prime}\right)+1$, which is seen to hold [ $\mu$ ] by virtue of the definitions of $n(\cdot, E), m\left(\cdot, E^{\prime}\right)$ and $E_{1}$.

The recurrence partition. The steps leading to the proof of the recurrencepartition theorem will be covered by a short sequence of lemmas. The purpose behind the lemma-approach is to indicate the relatively high level of generality of the component parts of the theorem and how, when they are assembled to form the complete theorem, the cumulative effect results in the necessarily sharpest possible form of the theorem.

We begin with an arbitrary but fixed measurable set $E \in \mathbb{S}$. Then except perhaps for a subset $Z$ of measure zero, the class $\mathbb{E}=\left\{E_{j}: j=1,2, \cdots\right\}$ covers $E$. Furthermore, any distinct pair $E_{j}, E_{k} \in \mathbb{E}, j<k$, is disjoint. For if $x \in E_{j} \cap E_{k}$, then by definition of $E_{j}, T^{i} x \in E$, but since $j<k$ this contradicts the fact that $k$ is the least integer such that $T^{k} x \in E$, which follows from the definition of $E_{k}$. Hence, we have

LEMma 4. Let $T$ be a measurable transformation. For every $E \in \subseteq$, the class $\mathbb{E}=\left\{E_{j}: j=1,2, \cdots\right\}$ partitions $E[\mu]$.

Now under the action of the measurable transformation $T$, and its iterates $T^{k}$ up to and including $j-1$, each $E_{j} \subset E, j>1$, is mapped into $E^{\prime}$. Furthermore, $T^{h} E_{j} \cap T^{i} E_{j}=\varnothing$ (the empty set) whenever $1 \leqq h<i \leqq j-1$. For if a point $y$ is in the intersection, then on the one hand since $y \in T^{h} E_{j}$, there exists 
an $x \in E_{j}$ such that $T^{h} x=y$. On the other hand, since $y \in T^{i} E_{j}$, it follows that $T^{j-i} y \in E$. Combining these facts, we have $T^{j-i}\left(T^{h} x\right)=T^{j-(i-h)} x \in E$, where $j-(i-h)<j$, thus contradicting the definitions of $n(\cdot, E)$ and $E_{j}$. These observations are summarized in:

LEMMA 5. Let $T$ be a measurable transformation and $E \in \mathbb{S}$. The sets of the class $\mathfrak{S}_{j}=\left\{T^{i} E_{j}: i=1, \cdots, j-1, j>1\right\}$ are pairwise disjoint, and each set of $\widetilde{S}_{j}$ is in $E^{\prime}$.

In general, the totality of sets in both $\mathfrak{S}_{j}$ and $\mathfrak{S}_{k}, j \neq k$, are not pairwise disjoint. This desirable property is secured if $T$ assumed to be one-to-one in addition to being measurable. For if under this additional assumption the elements $T^{h} E_{j}$ and $T^{i} E_{k}$ of $\mathfrak{S}_{j}$ and $\mathfrak{S}_{k}$ meet, we have the following two cases: (a) if $h=i$, then since $T$ is one-to-one $T^{-h}\left(T^{h} E_{j} \cap T^{h} E_{k}\right)=E_{j} \cap E_{k} \neq \varnothing$, which contradicts Lemma 4 ; (b) if $h<i$, then $T^{-h}\left(T^{h} E_{j} \cap T^{i} E_{k}\right)=E_{j} \cap T^{i-h} E_{k} \neq \varnothing$. But $k>m=i-h \geqq 1$, so that $T^{m} E_{k} \subset E^{\prime}$. This, with the fact that $E_{j} \subset E$, gives a contradiction. These observations, along with Lemma 5 yield

LEMMa 6. Let $T$ be a one-to-one measurable transformation. The union of the family $\left\{\mathfrak{S}_{j}: j=2,3, \cdots\right\}$ of classes induced by $T$ on $E \in \mathbb{S}$ is a class of pairwise disjoint subsets of $E^{\prime}$.

In general the class $\cup_{j=2}^{\infty} \mathfrak{S}_{j}$ does not cover $E^{\prime}$, i.e., the union of its members is generally a proper subset of $E^{\prime}$. However, $F=E^{\prime}-U_{j=2}^{\infty} \mathfrak{S}_{j}$ has the property that $T^{-1} F \subset F$, if $T$ is measurable. For if $y$ is a point in $T^{-1} F$ and also in the complement of $F$, i.e., in $E \cup \cup_{j=2}^{\infty} \Im_{j}$, then necessarily $T y \in F$ and $T y \in E \cup \cup_{j=2}^{\infty} \mathfrak{S}_{j}$ simultaneously, which is impossible $[\mu]$.

Now, our present aim is to insure that $F$ and $G=E \cup \cup_{j=2}^{\infty} \Im_{j}$ are invariant [ $\mu$ ] subsets of $X$. In view of the fact just proved, it is sufficient to require that $T$ be measure-preserving. For then, if $Z=F-T^{-1} F$, it follows that $\mu(F)=\mu\left(Z \cup T^{-1} F\right)=\mu(Z)+\mu\left(T^{-1} F\right)=\mu(Z)+\mu(F)$, which implies that $\mu(Z)$ $=0$, providing that $\mu(X)<\infty$ (the finiteness being needed to permit additive cancellation). Hence, under these additional conditions, $T^{-1} F=F[\mu]$. In like manner it may be shown that $T^{-1} G \supset G[\mu]$, and also that $T^{-1} G=G[\mu]$. Assembling these findings we have

LEMMA 7. Let $T$ be a measure-preserving transformation on a finite measure space. For every $E \in \subseteq$, the sets $G=E \cup \cup_{j=2}^{\infty} \mathfrak{S}$, and $F=G^{\prime}$ are invariant $[\mu]$ under $T$.

RECURRENCE-PARTITION THEOREM. Let $T$ be an invertible measure-preserving transformation on a finite measure space $(X, \mathfrak{S}, \mu)$. Then for every $E \in \mathfrak{S}$, the class $\mathfrak{B}=\left\{E, \bigcup_{j=2}^{\infty} \mathfrak{S}_{j}, F\right\}$ is a partition of $X$, and the sets $G=E \cup \cup_{j=2}^{\infty} \mathfrak{S}_{j}$ and $F=G^{\prime}$ are invariant $[\mu]$ under $T$.

Proof. Since $T$ is invertible (and hence measurability-preserving) the elements of the class $\mathfrak{B}$ are measurable. Furthermore, $\mathfrak{B}$ covers $X$. By Lemma 6 
the elements of $\bigcup_{j=2}^{\infty} \Im_{j}$ are pairwise disjoint and $\left(\cup_{j=2}^{\infty} \Im_{j}\right) \cap E=\varnothing$. By definition $F \cap G=\varnothing$. Finally, by Lemma $7, G$ and $F$ are invariant $[\mu]$ under $T$.

CoRollaRy. If $I \in \subseteq$ is invariant under Tand $E \subset I[\mu]$, then $E \subset G \subset I[\mu]$.

A brief comment on the hypothesis of the recurrence-partition theorem is in order. The requirement of the invertibility of $T$ may appear to be overly restrictive in view of the fact that none of the preceding lemmas on which the theorem depends requires such an assumption. However, the additional requirement is a consequence of the cumulative effect of the various hypotheses which are now required to hold simultaneously. In particular, Lemma 6 requires one-to-oneness of $T$ and Lemma 7 requires measure-preservation. A moment's reflection shows that these two requirements along with that of measurability-preservation of $T$ (in order to have elements of $\mathfrak{S}_{j}$ measurable) imply that $T$ is onto, and hence, invertible $[\mu]$. The corollary follows immediately from the properties of the $\mathfrak{\Im}_{j}$ families. Phrased equivalently, the corollary states that: $G$ is the (unique $[\mu]$ ) smallest $T$-invariant set containing $E$.

Exit function partitions. It has been stated that the primary object of study in this note is the recurrence partition. The exit function has been introduced simply to show that a natural complementary approach to the partition problem was available. Beyond this introduction, the two lemmas below, and a calculation of some $m$-integrals in the applications section that follows (Equations (11) and (12)), we shall not pursue the theory of the exit function further in this work. Specifically, we note, in view of the equations (7) and (8),

Lemma 8. Let $T$ be a measurable transformation. For every $E \in \mathbb{S}$ the class $\mathfrak{D}=\left\{D_{j}: j=1,2, \cdots\right\}$ partitions $E[\mu]$.

The proof is analogous to Lemma 4. Furthermore, we observe without proof

Lemma 9. Let $T$ be a measurable transformation. Let $\left\{E, E^{\prime}\right\}$ partition $X$, and let $\mathfrak{D}=\left\{D_{j}: j=1,2, \cdots\right\}$ and $\mathfrak{F}^{\prime}=\left\{E_{j}^{\prime}: j=1,2, \cdots\right\}$ be the associated exit function and recurrence function partitions of $E$ and $E^{\prime}$ respectively. Then for every integer $j \geqq 1$,

$$
T D_{j+1} \cup T E_{j+1}^{\prime}=D_{j}
$$

\section{Applications.}

RECURRENCE-INTEGRAL THEOREM. Let $T$ be an invertible measure-preserving transformation on a normalized finite measure space $(X, \mathfrak{S}, \mu)$. Let $n(\cdot, E)$ be the recurrence function for $E \in \subseteq$, and let $F \in \subseteq$ be the union of all $T$-invariant subsets in the complement of $E$. Then 


$$
\int_{E} n(\cdot, E) d \mu=1-\mu(F) .
$$

Proof. By Lemma 1, the recurrence function is measurable over $E$. By the recurrence-partition theorem the complement $E^{\prime}$ of $E$ may be represented as the union of a disjoint class of sets:

$$
E^{\prime}=F \cup \bigcup_{j=2}^{\infty} \Im_{j} .
$$

Since $\mu$ is countably additive and $T$ is measure-preserving,

$$
\mu\left(E^{\prime}\right)=\mu(F)+\sum_{j=2}^{\infty}(j-1) \mu\left(E_{j}\right) .
$$

By Lemma 4,

$$
\mu(E)=\sum_{j=1}^{\infty} \mu\left(E_{j}\right) .
$$

Hence

$$
\begin{aligned}
1=\mu(E)+\mu\left(E^{\prime}\right) & =\sum_{j=1}^{\infty} j \mu\left(E_{j}\right)+\mu(F) \\
& =\sum_{j=1}^{\infty} \int_{E_{j}} n(\cdot, E) d \mu+\mu(F) \\
& =\int_{E} n(\cdot, E) d \mu+\mu(F),
\end{aligned}
$$

and the theorem is proved.

CoRollary (KAC's TheOREM [1]). With the hypothesis as before, but now with $\mu(E)>0$ and $T$ ergodic,

$$
\int_{E} n(\cdot, E) d \mu=1
$$

Proof. By the recurrence partition theorem, the sets $G=E \cup \cup_{j=2}^{\infty} \mathfrak{S}_{j}$ and $F=G^{\prime}$ are $T$-invariant $[\mu]$. If $T$ is ergodic and $\mu(E)>0$, where $E \subset G$, then necessarily $\mu(F)=0$.

ERGODICITY THEOREM. Let $T$ be an invertible measure-preserving transformation on a finite measure space $(X, \mathfrak{S}, \mu)$, and let $\mathfrak{B}$ be the recurrence partition of $X$ induced by an arbitrary positive set $E$, with $\mu(F)=0$. A sufficient condition that $T$ be ergodic on $X$ is that $E$ contains no proper subsets with recurrent parts. 
Proof. Suppose $T$ is not ergodic. The existence of a proper subset of $E$ with a recurrent part is proved as follows: First, by nonergodicity, there exists a partition of $X$ into two proper $T$-invariant subsets of $X:\left\{I, I^{\prime}\right\}$. Since $\mu(F)=0, X=G \cup F=G[\mu]$. Hence $G=I \cup I^{\prime}[\mu]$. By the corollary of the recurrence-partition theorem these two sets partition $E$ into two proper positive subsets one which is $H=E \cap I$; so that $\mu(E)>\mu(H)>0$. By Lemma 4, $H$ may be represented as the following union of disjoint sets: $H=\bigcup_{j=1}^{\infty} H$ $\cap E_{j}[\mu]$. Since $H$ is positive and $\mu$ is countably additive, there exists an integer $k$ such that $H \cap E_{k}$ has positive measure. In view of the fact that $T^{k}\left(H \cap E_{k}\right) \subset E, H \cap E_{k}$ is the required recurrent part of $H \subset E$.

The condition of the preceding theorem is not necessary. Consider the unit shift transformation $T$ on the space $\left\{X_{6}=0,1, \cdots, 4\right\}$ of integers: $T x=x+1(\bmod 5) . T$ is invertible, measure-preserving and ergodic, when $\subseteq$ consists of all subsets of $X_{5}$ and when $\mu$ is additive such that $\mu(\{x\})=1 / 5$ for every singleton. However, each subset $E$ of more than one element contains proper subsets with recurrent parts (with respect to $E$ ).

The criterion given in the ergodicity theorem for the ergodicity of an invertible measure-preserving transformation may conceivably be of physical interest after suitable identifications of the abstract and concrete concepts have been made. Consider for example the pairing of a finite measure space $(X, \mathfrak{S}, \mu)$ with an experimental domain. Let some subset $E \in \mathfrak{S}$ be a region directly accessible to the experimental probes. The latter may be used to record the phenomenon of interest by means of photometers, neutron fluxmeters, magnetic flux-meters, heat flow-meters, etc. Thus $X$ is the domain over which the phenomenon occurs, $\subseteq$ is the class of all measurable subsets of $X$ with at least one positive member $E$ accessible to the probes, $\mu$ is the appropriate measure (volume-valued, mass-valued, energy-valued, etc.), and finally $T$ is the process governing the spatial or temporal evolution of the phenomenon of interest. The function $n(\cdot, E)$ then describes the recorded recurrence of the phenomenon in $E$ after the phenomenon has evolved in $X$. According to the ergodicity theorem and Equation (9), the ergodicity of the process $T$ may be determined by: (a) examining the directly accessible proper subsets of $E$ for recurrent parts and (b) by performing the calculation (9) using recorded $n(\cdot, E)$-values to determine $\mu(F)$. If no proper subsets of $E$ have recurrent parts and (9) shows that $\mu(F)=0$, then $T$ is ergodic. The importance of these results from the physical viewpoint lies in the fact that something may be deduced about the measure of a physically inaccessible possibly invariant subset $F$ of $X$ by suitable observations over a directly accessible subset $E$ of $X$ disjoint from $F$.

FIRST EXIT-INTEGRAL THEOREM. Let $T$ be an invertible measure-preserving transformation on a normalized finite space $(X, \mathfrak{S}, \mu)$. Let $E \in S$ be arbitrary and let $n(\cdot, E)$ and $m\left(\cdot, E^{\prime}\right)$ be the corresponding recurrence and exit functions of $E$ and $E^{\prime}$ respectively. Then 


$$
\int_{T\left(E-E_{1}\right)} m\left(\cdot, E^{\prime}\right) d \mu=\mu\left(E^{\prime}-F\right),
$$

where $F$ is the union of all T-invariant subsets of $E^{\prime}$.

Proof. By Lemma $2, m\left(\cdot, E^{\prime}\right)$ is measurable on $E^{\prime}$, and by Lemma 3

$$
\int_{E} n(\cdot, E) d \mu=\int_{E} \chi_{E-E_{1}} m\left(T(\cdot), E^{\prime}\right) d \mu+\mu(E)
$$

By the integral recurrence theorem,

$$
\int_{E-E_{1}} m\left(T(\cdot), E^{\prime}\right) d \mu=1-\mu(F)-\mu(E)=\mu\left(E^{\prime}-F\right) .
$$

Since $T$ is invertible and measure-preserving, the appropriate change of variables $T x \rightarrow x$ can be made in the integral over $E-E_{1}$ to get:

$$
\int_{T\left(E-E_{1}\right)} m\left(\cdot, E^{\prime}\right) d \mu=\mu\left(E^{\prime}-F\right)
$$

the desired result.

COROLlary. With the hypothesis as before, but now with $\mu(E)>0$ and $T$ ergodic,

$$
\int_{T\left(E-E_{1}\right)} m\left(\cdot, E^{\prime}\right) d \mu=\mu\left(E^{\prime}\right)
$$

More detailed integral theorems for the exit function may be deduced by using the recurrence partition of $X$ directly. As an illustration of such an application, consider the

SECOND EXIT-INTEGRAL THEOREM. Let $T$ be an invertible measure-preserving transformation on a normalized finite measure space $(X, \mathcal{S}, \mu)$. If $\left\{E, E^{\prime}\right\}$ is a partition of $X$, then

$$
\int_{E^{\prime}-F} m\left(\cdot, E^{\prime}\right) d \mu=\sum_{j=2}^{\infty} \frac{j(j-1)}{2} \mu\left(E_{j}\right),
$$

where $F$ is the union of all $T$-invariant subsets in $E^{\prime}$.

Proof. By the recurrence partition theorem, $E^{\prime}-F=U_{j=2}^{\infty} \mathfrak{S}_{j}$. The integral of $m\left(\cdot, E^{\prime}\right)$ over any image $T^{k} E_{j}$ under $T^{k}$ of $E_{j} \in \mathfrak{S}_{j}, k=1, \cdots, j-1, j \geqq 2$, is clearly

$$
\int_{T^{k} B_{j}} m\left(\cdot, E^{\prime}\right) d \mu=(j-k) \mu\left(E_{j}\right)
$$


which follows from the definition of $\mathfrak{S}_{j}$ and the measure-preservation of $\mu$. Hence for $1 \leqq k \leqq j-1$,

$$
\int_{\mathfrak{S}_{j}} m\left(\cdot, E^{\prime}\right) d \mu=\frac{j(j-1)}{2} \mu\left(E_{j}\right),
$$

and (13) follows.

The preceding method of evaluating the integral of $m\left(\cdot, E^{\prime}\right)$ in terms of the partition $E$ of $E$ (Lemma 4) can be generalized to a method of evaluating the integral over $X$ of any integrable function on $X$. We summarize this method in the

GENERAL INTEGRAL THEOREM. Let $f$ be integrable on a finite measure space $(X, \mathfrak{S}, \mu)$. If $T$ is any invertible measure-preserving transformation on $X$, let $f_{k}(x)=f\left(T^{k} x\right)$ define the functions $f_{k}, k=0,1,2, \cdots$. Then, using the notation of the recurrence partition $\mathfrak{B}$ :

$$
\int_{X} f d \mu=\sum_{j=1}^{\infty} \int_{E_{j}} \sum_{k=0}^{j-1} f_{k} d \mu+\int_{F} f d \mu .
$$

In particular, if $T$ is ergodic and $\mu(E)>0$, then the last integral on the right in (14) vanishes and the problem of the integration of $f$ over $X$ reduces to one over $E$. One's interest in this theorem and all those above should increase directly with the measure of the complement of the positive set $E$.

Generalizations. There are several directions in which the results of the present study may be generalized. An immediate generalization of the recurrence function, for example, may be of the following kind. Let $E, G \in \mathbb{S}$ be disjoint. Let $n(x, E, G), x \in E$ be the least integer $n$ with the properties that (a) $T^{n} x \in E$, (b) $T^{i} x \in G, 1 \leqq j<n$. In this generalization the complement $E^{\prime}$ of $E$ has been replaced by an arbitrary proper subset $G$ of $E^{\prime}$. The recurrencepartition theorem may then be extended mutatis mutandis to $E, G$ and an invariant subset $F$ of $G$. The proof of this generalized partition theorem goes through as before; and in particular the generalized recurrence-integral theorem takes the form

$$
\int_{E} n(\cdot, E, G) d \mu=\mu(E \cup(G-F)),
$$

which reduces to (9) if $G=E^{\prime}$.

Acknowledgment. The authors take this opportunity to express their thanks to the reviewer for bringing to their attention an interesting paper by Shu-Teh Chen Moy [3], in which the concept of recurrence time is generalized to include sequences of successive returns of the wandering point to the basic set $E$. Kac's theorem in his classical form is shown to hold for each member of the generalized recurrence functions. The approach of Moy's paper 
is statistical and its results intersect those of the present paper at the point occupied by the classical form of Kac's theorem (Corollary to the Recurrence Integral Theorem in the present study).

\section{REFERENCES}

1. M. Kac, On the notion of recurrence in discrete stochastic processes, Bull. Amer. Math. Soc. vol. 53 (1947) pp. 1002-1010.

2. P. R. Halmos, Lectures on ergodic theory, Publications of the Mathematical Society of Japan, No. 3, Tokyo, Mathematical Society of Japan, 1956, pp. 5-9.

3. Shu-Teh Chen Moy, Successive recurrence times in a stationary process, Ann. Math. Statist. vol. 30 (1959) pp. 1254-1257.

General Dynamics Corporation, San Diego, California 